\title{
Finanzkrise, Finanzialisierung und Vergleichende Kapitalismusforschung
}

Der Beitrag erklärt die Entstehung und die unterschiedlichen Auswirkungen der Finanzkrise unter Rückgriff auf etablierte Theorien der Internationalen Politischen Ökonomie. Er greift für eine intertemporale Perspektive auf das FinanzialisierungsKonzept zurück sowie für die länderbezogene Perspektive auf das Theorieprogramm der Vergleichenden Kapitalismusforschung. In intertemporaler Perspektive zeigt der Beitrag, dass die Entstehung und das Ausmaß der Finanzkrise nicht ohne die grundlegenden Finanzialisierungsprozesse der letzten dreißig Jahre zu erklären ist. Mit Finanzialisierung wird ein Prozess bezeichnet, bei dem sich der Anteil der Profite und Haushaltseinkommen zwischen Produktions- und Finanzsektor deutlich zugunsten des Letzteren verschiebt und der zugleich zu einer entsprechenden Machtverschiebung zwischen den beiden Sektoren geführt hat. In international vergleichender Perspektive erklärt der Beitrag die stärkeren Auswirkungen der Krise in den angelsächsischen Ökonomien aus der zentralen Rolle der Kapitalmärkte bei der Unternehmensfinanzierung in diesen liberalen Marktwirtschaften und kontrastiert diesen Befund mit den relativ geringen Konsequenzen für die klassischen Hausbanken im Rheinischen Kapitalismus.

\section{Einleitung ${ }^{1}$}

Die Entstehung und der Verlauf der Finanzkrise können aus verschiedenen Perspektiven der Internationalen Beziehungen betrachtet werden, beispielsweise als Frage der zwischenstaatlichen Politikkoordination, des europäischen Mehrebenenregierens, der Wirkung internationaler Institutionen, oder auch der Konsequenzen für die globale Machtbalance. Ganz besonders interessant ist jedoch eine Analyse aus der Perspektive der Internationalen Politischen Ökonomie, verfügt diese doch über Konzepte, die sich nicht nur mit der politischen Krisenbearbeitung beschäftigen, sondern auch die ökonomischen Ursachen der Krise einbeziehen, insbesondere durch ihre Offenheit für die gegenseitige Befruchtung mit der Wirtschaftssoziologie sowie den heterodoxen Ansätzen der Wirtschaftswissenschaft.

1 Dieser Beitrag entstand im Rahmen eines Aufenthaltes am Max-Planck-Institut für Gesellschaftsforschung, dem ich für seine großzügige Unterstützung sehr verbunden bin. Für vielfältige Anregungen danke ich - neben den beiden anonymen Gutachtern oder Gutachterinnen der ZIB - Daniel Seikel, der Arbeitsgruppe Internationale Politische Ökonomie an der Goethe-Universität sowie den Mitgliedern des Netzwerkes Politische Ökonomie der globalen Finanzialisierungsprozesse, insbesondere Hans-Jürgen Bieling, Dorothee Bohle, Marcel Heires, Stefanie Hiß, Oliver Kessler, Daniel Mügge, Thomas Sablowski und Brigitte Young. Christian Möller, Max Breuer und Brigitte Holden haben mir bei der Erstellung des Manuskripts sehr geholfen. 
Dieser Beitrag versucht, die Entstehung und die unterschiedlichen Auswirkungen der Finanzkrise unter Rückgriff auf etablierte Theorien der Internationalen Politischen Ökonomie zu erklären. Dabei greift er einerseits auf das »Finanzialisierungs«Konzept (intertemporale Perspektive) und anderseits auf das Theorieprogramm der Vergleichenden Kapitalismusforschung (länderbezogene Perspektive) zurück. Keine der beiden Theorien liefert für sich allein eine befriedigende Erklärung - der Finanzialisierungs-Perspektive fehlt es an nationaler und internationaler Differenziertheit, der Vergleichenden Kapitalismusforschung an einer dynamischen bzw. historischen Perspektive, aber ihre Kombination erlaubt eine Reihe von Einsichten, die auch durch die fortschreitende Entwicklung der Krise nicht gleich relativiert werden. Gleichzeitig sind sie in ihrer Grundausrichtung kompatibel, insofern beide davon ausgehen, dass es nicht »den« Kapitalismus gibt, sondern dieses Wirtschaftssystem immer nur in seiner konkreten räumlichen und zeitlichen Einbettung angemessen analysiert werden kann.

Die Vorgehensweise dieses Beitrags strebt keine vollständige Erklärung der Krise, insbesondere ihres konkreten Verlaufs an (siehe zu Letzterem aber die Beiträge von Bieling und Kessler), im Vordergrund steht vielmehr die Identifikation der groben historischen und räumlichen Entwicklungen. Die grundlegende Vorgehensweise ist vergleichend: Zunächst wird das Finanzialisierungskonzept vorgestellt (Abschnitt 2.1) und darauf aufbauend in intertemporal vergleichender Perspektive erklärt, warum die strukturellen Veränderungen seit den 1970er Jahren die aktuelle Finanzkrise möglich gemacht haben (Abschnitt 2.2). Im zweiten, inter-nationalen Schritt werden kurz die Auswirkungen der Finanzkrise auf verschiedene nationale Wirtschaftssysteme verglichen (Abschnitt 3.1) und unter Rückgriff auf Ansätze der Vergleichenden Kapitalismusforschung die entsprechende Varianz erklärt (Abschnitt 3.2). Abschließend werden kurz einige Forschungsperspektiven skizziert, die sich aus diesen Überlegungen ergeben (Abschnitt 4).

\section{Finanzialisierung und die Finanzkrise in inter-temporaler Perspektive}

\subsection{Das Zeitalter der Finanzialisierung}

Bevor nachhaltige politische Antworten auf die globale Finanzkrise zu erwarten sind, bedarf es einer grundlegenden Reflexion darüber, welche Veränderungen und Dynamiken der letzten Dekaden die heutige Krise ermöglicht haben. Die Finanzkrise hat daher in jüngster Zeit das Interesse an der historischen Entwicklung des Kapitalismus stimuliert. Auch wenn in dieser Hinsicht vielfältige Kategorien koexistieren, besteht doch relativ weitgehende Einigung darüber, dass die Periode bis zu Beginn der 1970er Jahre in den westlichen Industrieländern durch ein relativ stabiles Produktionsmodell gekennzeichnet war (Fordismus). Die Periode des Fordismus zeichnete sich durch eine relativ standardisierte Massenproduktion für Massenbedarf auf der Grundlage von an Produktivitäts- und Preisentwicklung gekoppelten Löhnen aus, die zwischen Großunternehmen, gut organisierten Gewerkschaften und dem keynesianischen Staat 
jeweils auf nationaler Basis ausgehandelt wurden. Abgesichert wurde dieses Modell durch relativ stark kontrollierte nationale Finanzmärkte und Restriktionen für länderübergreifende Finanzflüsse. Es geriet seit Ende der 1960er Jahre zunehmend unter Druck und wurde durch den Zusammenbruch des Bretton Woods-Systems erheblich geschwächt (Andrews 2008).

Die Charakterisierung des Fordismus ist in der Literatur zwar weitgehend unstrittig, zugleich konkurrieren allerdings verschiedene Konzepte zur Analyse der ihm folgenden Produktionsweise. In den letzten Jahren hat eine Gruppe von ÖkonomInnen und SozialwissenschaftlerInnen eine Periodisierung der kapitalistischen Entwicklung nach den frühen 1970er Jahren mit dem Begriff der Finanzialisierung (financialization) untersucht. Damit wird ein Prozess bezeichnet, bei dem zunehmende Anteile der Unternehmensgewinne und Haushaltseinkünfte aus finanziellen Aktivitäten erzielt werden, im Vergleich zu jenen Anteilen, die sich direkt aus der Produktion bzw. Lohnarbeit ergeben. Damit einher geht eine Machtverschiebung sowohl innerhalb von Unternehmen als auch zwischen ihnen, bei der der Finanzsektor dem produzierenden Sektor übergeordnet wird. Diese Perspektive stellt traditionelle Sichtweisen des Kapitalismus, wonach der Finanzsektor - sowohl innerhalb einzelner Betriebe als auch im Kapitalismus generell - zur Unterstützung der »Realökonomie« diene, auf den Kopf. Hinzu kommt, dass sich Schwankungen und Krisen im Finanzsystem durch die engere Bindung der Unternehmen an den Finanzsektor viel stärker und unmittelbarer auf den produktiven Sektor auswirken (Windolf 2005 b: 52). Zwar hat es auch früher Finanzblasen und spekulative Perioden gegeben (zumeist von kürzerer Dauer, vgl. Kindleberger 1978), inzwischen hat sich der Finanzsektor jedoch in viel größerem Maße verselbständigt und eine ungewöhnliche Dominanz über den Rest der Wirtschaft erworben - wobei offen bleibt, inwiefern sich die gegenwärtige Finanzkrise letztlich existenzbedrohend auf diese Kapitalismusformation auswirken wird.

Da die neue Phase der Finanzialisierung unabhängig voneinander in verschiedenen Disziplinen und nationalen Kontexten aufgegriffen wurde, gibt es keine einheitliche Definition. So konkurrieren die regulationstheoretischen Konzeptionen eines finanzgeführten (Boyer 2000) bzw. finanzdominierten (Stockhammer 2007) Akkumulationsregimes mit Definitionen von post-keynesianischen Ökonomen:»Financialization refers to the increasing importance of financial markets, financial motives, financial institutions, and financial elites in the operation of the economy and its governing institutions, both at the national and international level « (Epstein 2001: 1; siehe auch Palley 2007: 3). Gleichzeitig wird aus der Sicht einer kulturellen politischen Ökonomie - also einer Perspektive, die kulturellen Faktoren wie Identitäten, Deutungen und Ritualen auch im Studium der politischen Ökonomie mehr Geltung verschaffen möchte (Best/Paterson 2009) - zunehmend die prägende Kraft der Kapitalmärkte im Alltagsleben betont (Leyshon/Thrift 2007; Langley 2008; Erturk et al. 2008). ${ }^{2}$ Sinnvoll ist jedenfalls die Differenzierung von zwei Komponenten von Finanzialisierung,

2 Eine alternative Verwendung des Begriffs der Finanzialisierung im deutschsprachigen Raum bezeichnet, »wie die Zwänge und Strukturen eines Finanzmarkt-Regimes in die internen Organisationsstrukturen der Unternehmen übersetzt werden« (Windolf 2005 b: 17). 
nämlich einerseits die Verschiebung des Anteils an den Profiten zwischen Produktions- und Finanzsektor und andererseits die Machtverschiebung zwischen den beiden Sektoren (Nölke und Perry 2007: 4-6). Solche Machtverschiebungen gab es bereits zu früheren Zeiten, worauf z. B. Hilferding (1910) in seinen Studien zum Finanzkapital hingewiesen hat (vgl. auch Arrighi 1994), doch gehen die Entwicklungen der letzten drei Dekaden deutlich über diese historischen Vorläufer hinaus. Zudem unterscheidet sich der Begriff der Finanzialisierung auch vom etwas engeren Konzept des Finanzmarkt-Kapitalismus (Windolf 2005 a), bei dem die - über institutionelle Investoren und Finanzanalysten vermittelte - Dominanz der Finanzmärkte über produzierende Unternehmen im Vordergrund steht, während die Transformation und Profitsteigerung des Finanzsektors selbst sowie die zunehmende finanzielle Durchdringung des Alltagslebens weniger untersucht werden.

Der erste, eher strukturelle Teil der Definition lässt sich sehr gut anhand einiger Daten illustrieren, die demonstrieren, dass sich die Anteile des Finanzsektors an den Gewinnen der US-Ökonomie in den vergangenen 30 Jahren mehr als verdoppelt haben.

\section{Abbildung 1: Anteil des Finanzsektors am US-Gesamtprofit}

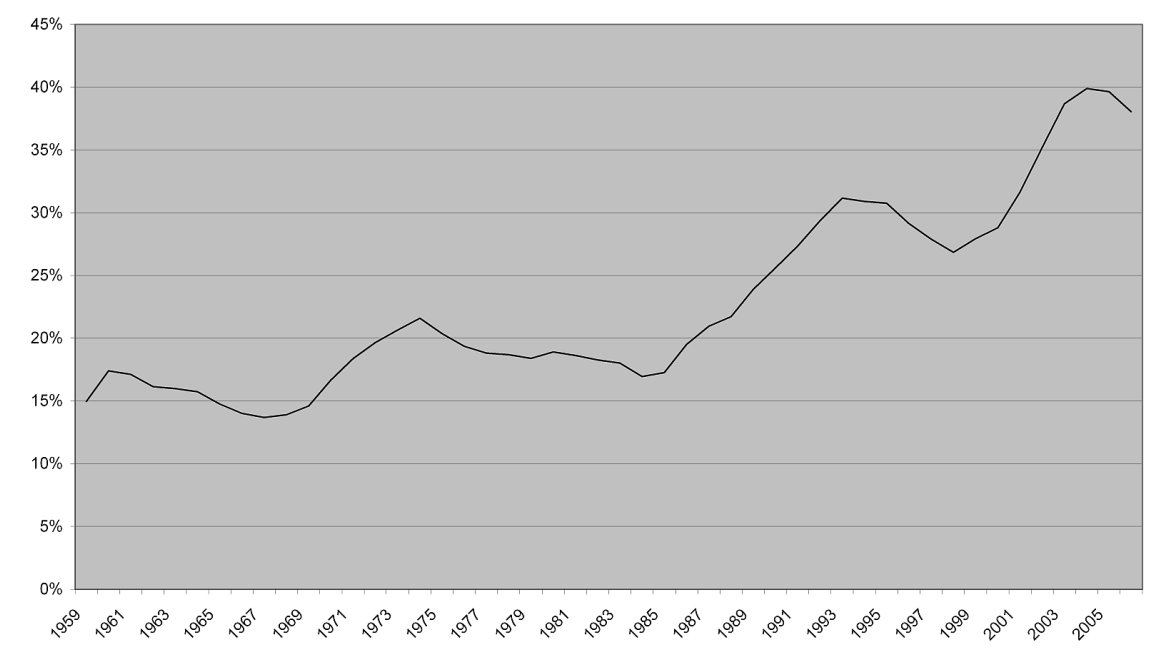

Quelle: President of the United States of America (2008: 332).

Weiterhin hat sich nicht nur - nach den aktuell verwendeten Rechnungslegungsstandards - die Profitabilität des Finanzsektors besser entwickelt als jene der meisten produktiven Sektoren, sondern auch innerhalb von Unternehmen des produktiven Sektors ist der Anteil der Profite aus Finanzmarktaktivitäten deutlich gestiegen (Crotty 2002: 34-36; Krippner 2005) - einige dieser Unternehmen haben inzwischen grö- 
ßere Einnahmen aus Finanztransaktionen als aus ihrer eigentlichen Produktion, wie in Deutschland am Beispiel der Porsche AG und ihren Gewinnen durch Transaktionen mit VW-Aktien zuletzt sehr deutlich wurde. Ermöglicht wurde diese Profitsteigerung durch eine große Anzahl von finanziellen Innovationen wie Derivaten und der Verbriefung von Krediten, die insbesondere von Investmentbanken vorangetrieben wurden. Während zu Beginn des Finanzialisierungsprozesses noch die Unternehmensfinanzierung im Vordergrund stand, dehnte sich diese - auch auf Grund entsprechender politischer bzw. rechtlicher Anreize - in den letzten Jahren auch auf andere Sektoren aus, einschließlich der Konsumgüter- Studienkredit- und Immobilienfinanzierung (Montgomerie 2006), wobei Letztere ein besonders großes Volumen annahm. Hypotheken wurden insbesondere in den USA zunehmend verbrieft. Die entsprechenden Ausfallrisiken wurden damit vom unmittelbaren Finanzier auf die Abnehmer der Verbriefungen verlagert. Damit einher ging eine deutliche Ausweitung jener Bevölkerungsschichten, die überhaupt Wohneigentum erwerben konnten. Die entsprechende Nachfragesteigerung führte zudem auch zu einer Wertsteigerung von Wohneigentum, welche über die Erhöhung von Hypotheken auch zur Konsumfinanzierung genutzt wurde. Die Ausweitung des Immobilienfinanzierungsmarktes erlangte durch Finanzinnovationen ein Momentum, das sich letztlich dann auch als strukturelle Voraussetzung der Subprime-Krise erwies (siehe den Beitrag von Young sowie Schwartz/ Seabrooke 2008).

Der zweite Teil der Definition - die Machtverschiebung zwischen produktivem und Finanzsektor - lässt sich zwar weniger gut messen, jedoch insofern sehr gut veranschaulichen, als die entsprechende Auseinandersetzung diskursiv unter dem Schlagwort des shareholder value geführt wurde (Froud et al. 2000). Im Vordergrund steht hier die Umgestaltung der Unternehmenskontrolle zugunsten von - zumeist eher kurzfristig orientierten - Finanzmarktakteuren (Overbeek et al. 2007). Während in dieser Diskussion zunächst die Rolle von Pensionsfonds und anderen institutionellen Investoren im Vordergrund stand (Useem 1996; Nölke 1998; Crotty 2002; Windolf 2005 a), verlagerte sich in jüngerer Zeit die Aufmerksamkeit auf Hedgefonds und private equity-Unternehmen. $\mathrm{Zu}$ den einzelnen Instrumenten der shareholder valueAgenda gehören unter anderem Aktienoptionspläne (um die Interessen der Manager an die Interessen der Finanzmarktakteure zu koppeln), die Schaffung eines aktiven Markts für Unternehmenskontrolle (zur Disziplinierung erfolgloser Manager) und Aktienrückkaufprogramme, bei denen die Gewinne der Unternehmen nicht reinvestiert, sondern zugunsten der Erhöhung der Aktienkurse verwendet werden. Umstritten ist allerdings noch, inwieweit die shareholder value-Agenda von den genannten Finanzmarktakteuren mit direkten Einzelfallinterventionen durchgesetzt wird, oder ob eher die damit verbundenen institutionellen Mechanismen (z. B. Aktienoptionsprogramme) ausschlaggebend sind (Kädtler/Sperling 2001; Kädtler 2005). Weiterhin beschränkt sich Finanzialisierung nicht nur auf den Kernbereich der Unternehmensfinanzierung, sondern erfasst in Form einer Masseninvestitionskultur (Erturk et al. 2005) auch einzelne Haushalte, insofern diese im Rahmen ihrer Rentenfinanzierung nach der Erosion der staatlichen Altersversorgung in zunehmendem Maße von Finanzmärkten, insbesondere den genannten Strategien von institutionellen Investoren, 
abhängen. Auch bei der Immobilienfinanzierung auf Kredit zeigte sich insbesondere in den USA eine erhebliche Verschiebung der relativen Anteile von Lohnarbeit und finanziellen Aktivitäten an den Haushaltseinkommen, zumindest in einer Phase permanent steigender Immobilienpreise.

Auch wenn der Prozess der Finanzialisierung von technologischen Faktoren, wie zum Beispiel im Bereich der Telekommunikation und der Finanzmathematik sowie durch demographische Entwicklungen unterstützt wurde, wird deutlich, dass Finanzialisierung keine bloß strukturelle Entwicklung des Kapitalismus darstellt, sondern auch ein politisch angeleitetes und vorangetriebenes Projekt ist. Als wesentliche Ursache des Übergangs zur Finanzialisierung sind zunächst die Liberalisierung des internationalen Kapitalverkehrs nach Ende des Systems von Bretton Woods 1973 zu nennen sowie die nachfolgenden Maßnahmen zur Reform der nationalen Kapitalmärkte, vor allem in den USA und Großbritannien (siehe den Beitrag von Bieling sowie Helleiner 1994; Abdelal 2007), auch wenn die ersten Grundlagen bereits durch die Tolerierung der kaum regulierten Euromärkte in den 1960er Jahren gelegt wurden (Burn 1999; Konings 2008). In den 1980er und 1990er Jahren gewann der Prozess der Finanzialisierung erheblich an Schwung, insbesondere nachdem die von Greenspan geführte US-Notenbank die von seinem Vorgänger Volcker gewählte Hochzinspolitik revidierte und damit die Aufnahme von Krediten für Finanzmarktspekulationen erleichterte; schon der »Volcker-Schock« hatte allerdings dazu beigetragen, Geld von der Realwirtschaft in den Finanzsektor zu transferieren (Konings 2008: 49). Zudem wird der Finanzialisierungsprozess in nicht unerheblichem Maße von einem deutlichen Überschuss an Geldvermögen auf der Suche nach rentablen Anlagemöglichkeiten vorangetrieben. Dieser Ersparnisschwemme (savings glut) ist auch eine Folge von Vermögensakkumulation und Nachfrageausfall durch soziale Segmentierung in den westlichen Gesellschaften (Deutschmann 2008: 16). Wissenschaftlich unterstützt durch den Aufstieg der Chicago-Schule in den Wirtschaftswissenschaften haben insbesondere die Reagan- und Thatcher-Regierungen hier wesentliche Weichenstellungen vorgenommen, die weder von der Blair- noch der Clinton-Administration revidiert wurden - im Gegenteil, ist Letztere doch unter anderem für die Revision des Glas-Steagall Act und damit für die Aufweichung der Trennung zwischen Investment- und Geschäftsbanken verantwortlich. Finanzialisierung war eine wesentliche Komponente des neoliberalen Politikprogramms, wenn auch Neoliberalismus und Finanzialisierung nicht deckungsgleich sind; Ersterer umfasst ja zudem auch Fragen der Handelsliberalisierung und der Privatisierung (Cerny 2008; Lee Mudge 2008). ${ }^{3}$ Auch die Europäische Union - und hier besonders die Europäische Kommission - hat den Prozess der Finanzialisierung der europäischen Volkswirtschaften nachhaltig unterstützt, insbesondere im Bemühen um die Schaffung eines paneuropäischen Finanzmarktes (Bieling 2005; Windolf 2005 b). Schließlich kann sich das Bemühen um eine Intensivierung von Finanzialisierung auf eine breite gesellschaft-

3 Finanzialisierung ist auch nicht deckungsgleich mit der Globalisierung der Waren- und Dienstleistungsproduktion, sondern kann empirisch und theoretisch getrennt analysiert werden (vgl. Krippner 2005: 193-198). 
liche Basis stützen, insofern nicht zuletzt die Rentierinteressen der westlichen Mittelschichten - als Erben oder als Anteilseigner von Pensionsfonds - von der Privilegierung des Finanzsektors profitieren und - auf einem extrem kompetitiven Markt ihre Fonds zu einer Maximierung von Renditen antreiben (Deutschmann 2008).

Die Finanzialisierung der Wirtschaft in den letzten Dekaden hatte deutliche Vorzüge, aber auch erhebliche Nachteile. Zu den Vorzügen gehört zunächst eine erhebliche Nachfragestimulierung, die - insbesondere in den USA - von der Wertsteigerung auf Aktienmärkten sowie von Wohnimmobilien und deren Nutzung für die Konsumfinanzierung ausging und als einer der wesentlichen Gründe für das relativ hohe Wirtschaftswachstum in den hochgradig finanzialisierten Volkswirtschaften Großbritanniens und der USA gilt (Stockhammer 2007: 8-12). Sie ist damit zugleich die Antwort des Neoliberalismus auf die Notwendigkeit keynesianischer Nachfragestimulierung (siehe den Beitrag von Young in diesem Forum). Zudem konnten nun Bevölkerungsteile Wohneigentum erwerben, denen diese Option zuvor nicht offen stand. Die konventionelle ökonomische Theorie hob zudem die effizienzsteigernde Wirkung der Finanzialisierung hervor, auch wenn dieses Argument im Kontext des Aufstiegs der behavioral finance-Literatur und ihrer Betonung irrationalen Verhaltens auf den Finanzmärkten weniger prominent geworden ist (Palley 2007: 4-6).

$\mathrm{Zu}$ den Nachteilen der Finanzialisierung gehört zunächst die Verschiebung der Einkünfte vom Produktionssektor zum Finanzsektor sowie innerhalb von Unternehmen von der Produktion zu Finanzaktivitäten, was - zusammen mit dem gewandelten Kräfteverhältnis zwischen Kapital und Arbeit - zu einer erheblichen Reduktion des Anteils der Löhne am gesellschaftlichen Einkommen geführt hat. Dementsprechend konnte inzwischen nachgewiesen werden, dass Finanzialisierung ganz erheblich zu Einkommensungleichheiten beigetragen haben, trotz (bzw. wegen) der Ausweitung des Anteils von Bevölkerungsschichten mit Immobilienbesitz und Anteilen an Investmentfonds (Palley 2007: 10-14). Damit einher geht ein politischer Effekt, insofern diese »Eigentümergesellschaft« tendenziell politisch eher konservativen Positionen zuneigt (Deutschmann 2008; Schwartz/Seabrooke 2008).

Auch die volkswirtschaftlich positive Bewertung des Finanzialisierungsprozesses ist umstritten. Mit Ausnahme der USA und ihrer geringen Sparquote hat dieser Prozess eher wachstumsbremsend gewirkt, da Rentiers über eine geringere Konsumneigung als Arbeiter verfügen (vgl. van Treeck et al. 2007: 640). Weiterhin besteht ein deutliches Spannungsverhältnis zwischen Finanzialisierung und Investitionen im Produktionssektor, durch entsprechende Anreizstrukturen für das Management, Verknappung interner Finanzressourcen, erhöhter Unsicherheit und Verkürzung des Kalkulationshorizonts (Crotty 2002; Orhangazi 2006; Stockhammer 2007). Schließlich ist Finanzialisierung nicht nur - wegen deregulierten Finanzmärkten, der Zunahme grenzüberschreitender Finanzflüsse und flexiblen Wechselkursen - wesentlich volatiler, sondern auch krisenanfälliger als das frühere fordistische Produktionsmodell, was sich nicht zuletzt an der Häufung von Finanzkrisen in den letzten Jahren gezeigt hat - und unter anderem auch dazu geführt hat, dass einige der neuen Immobilienbesitzer inzwischen hoch verschuldet sind und zudem ihre Häuser wieder verloren haben 
(Emunds 2008: 464), was die Fragilität des mit der Finanzialisierung einhergehenden Nachfrageschubes verdeutlicht.

\subsection{Finanzkrise und Finanzialisierung}

Generell ist noch nicht systematisch geklärt, welchen Anteil der Finanzialisierungskapitalismus am Ausbruch und an den Dimensionen der aktuellen Finanzkrise hat. Grundsätzlich gehe ich jedoch davon aus, dass das Ausmaß der aktuellen Finanzkrise ohne den Prozess der Finanzialisierung nicht denkbar ist. Die aktuelle Krise hätte unter den Bedingungen des Fordismus mit seinen hoch regulierten Finanzmärkten nicht entstehen können. Das gilt sowohl für die Entstehung der Krise in den USA als auch für ihre Weiterverbreitung zwischen hochgradig verflochtenen nationalen Finanzmärkten. Dementsprechend gab es vor Beginn der Finanzialisierung jahrzehntelang kaum größere Finanzkrisen (1930er bis 1960er Jahre), während sich deren Frequenz und Dimension in jüngster Zeit so stark erhöht haben (»Start up«-Krise, Russland-Krise, Ostasien-Krise etc.), dass manche Beobachter inzwischen von einer finalen Krise des Finanzialisierungskapitalismus sprechen (Blackburn 2008; Foster 2008; siehe auch den Beitrag von Bieling in diesem Heft). Dementsprechend kann man argumentieren, dass die Finanzialisierungsdynamik - zumindest in hochgradig finanzialisierten Wirtschaften wie den USA - die börsennotierten Unternehmen des produzierenden Sektors tendenziell in die Krise führt, da die von den Finanzmärkten zuletzt erwarteten 12-15\% return auf die Kapitalkosten langfristig allenfalls von Unternehmen in oligopolistischen Märkten oder mit hochgradig spekulativen und riskanten Strategien erzielt werden können - oder mit betrügerischen Praktiken wie im Fall von Enron und Worldcom (Froud et al. 2000: 106-7; Crotty 2002: 30-34; Windolf 2008: 10-13).

Weiterhin ist die Finanzkrise direkt im Zusammenhang mit einem typischen Produkt der Finanzialisierung entstanden: durch die Transformation von konventionellen, durch einen greifbaren Gegenwert gesicherten Hypotheken in anonymisierte, verbriefte Finanzprodukte (siehe die Beiträge von Bieling und Kessler in diesem Heft). Konventionelle Hypotheken, wie sie in Kontinentaleuropa üblich sind, werden von der Krise nicht direkt berührt. Darüber hinaus sind finanzialisierte Volkswirtschaften wesentlich stärker von Boomphasen und den folgenden Krisen geprägt als herkömmliche Systeme, da sich Aktien und Finanzprodukte besser zur Spekulation eignen als Unternehmen und Immobilien (Emunds 2008: 461). Weitergehend kann man sogar argumentieren, dass der Zyklus von »boom and bust« einen notwendigen Bestandteil der Finanzialisierung darstellt: Um in finanzialisierten Wirtschaften Wachstum zu schaffen, werden immer wieder neue Anlageperspektiven benötigt. Bei jeder neuen Anlageperspektive steigert sich die Dynamik der Finanzmärkte so sehr, bis kaum noch ein Bezug zu den ursprünglich vorhandenen realwirtschaftlichen Veränderungen besteht. Diese dynamische Entwicklung geht mit relativ hohen Wachstumsraten und der Steigerung von Vermögenswerten einher, endet jedoch regelmäßig irgendwann in einer krisenhaften Entwicklung, bei der die geschaffenen Werte leicht 
wieder zerstört werden können (Emunds 2008: 462). Nur dem - von der US-Notenbank aktiv unterstützten - reibungslosen Übergang der Technologieaktien-Blase in die Immobilien-Blase hat es die US-Wirtschaft zu verdanken, dass sie nicht bereits zu Beginn der 1990er Jahre in eine größere Krise abgeglitten ist. Zudem verhindern die im Vergleich zu den 1930er Jahren wesentlich höhere Staatsquote sowie der ausgebaute Wohlfahrtsstaat, dass die durch Finanzialisierung ausgelösten Rezessionen - in den westlichen Ökonomien - nicht harscher ausfallen (Stockhammer 2007: 21).

Grundsätzlich ist also die Phase des Finanzialisierungskapitalismus krisenanfälliger als der vormalige Fordismus. Für einen Finanzialisierungs-Theoretiker ist die Subprime-Krise dann auch keine Überraschung und wurde bereits seit einiger Zeit erwartet:

»The stability of an equity-based regime depends on monetary policy which controls financial bubbles and thus the diffusion of finance may push the economy into a zone of structural instability. The next major crisis may originate in the USA whose economy approximates most closely to the model« (Boyer 2000: 111).

Jene politischen Maßnahmen, die zur Vertiefung des Finanzialisierungs-Modells dienen sollten, gehören nun auch zu den Faktoren, die die Krise schließlich intensiviert haben. Neben den oben genannten Maßnahmen der Finanzmarktliberalisierung gehörte dazu auch die Umstellung von Rechnungslegungsstandards von historischen Anschaffungswerten zur aktuellen Marktbewertung (fair value oder mark-to-market accounting). Diese Umstellung ist maßgeblich durch den Übergang zur Finanzialisierung bedingt worden und trägt zur weiteren Finanzialisierung bei (Perry und Nölke 2006; Nölke und Perry 2007), gehört nun jedoch zu den ersten Faktoren, die im Rahmen der Krisenbekämpfung zumindest teilweise wieder korrigiert worden sind: Während die Bewertung von Finanzgütern zu Marktpreisen in einer Aufschwungperiode - im Vergleich zur Bewertung nach den historischen Anschaffungspreisen - zu einer höheren Bewertung dieser Güter führt (und damit zur oben dokumentierten Steigerung des Anteils von Finanzgütern an den Gewinnen), führt die gleiche Bewertung in einer Abschwungphase zu einer niedrigeren Bewertung dieser Güter und intensiviert damit die Krise von Banken und anderen Finanzmarktakteuren ganz erheblich, zumal diese Akteure durch die Wertberichtigungen und damit einhergehenden Verluste zu Verkäufen gezwungen werden, die wiederum zu einem weiteren Preisverfall führen. Auch dieser Zusammenhang ist bereits vor Ausbruch der Finanzkrise klar artikuliert worden:

»When liquidity plays an important role as in times of financial crisis, asset prices in some markets may reflect the amount of liquidity available in the market rather than the future earning power of the asset. Mark-to-market accounting is not a desirable way to assess the solvency of a financial institution in such circumstances [...] In contrast, if historical cost accounting is used, banks are allowed to continue and can meet all their future liabilities« (Allen/Carletti 2006: 1).

Die im Rahmen der Finanzkrise entschiedene selektive Abkehr von der mark-tomarket-Bewertung adressiert dieses Problem, bleibt aber zunächst bescheiden und ist bei den an der Konsistenz ihrer Regelsysteme interessierten Institutionen der Rech- 
nungslegungsstandardisierung (zum Beispiel dem International Accounting Standards Board) sehr umstritten.

Schließlich lassen sich auch die Dimensionen der Finanzkrise nur verstehen, wenn die im Zusammenhang der Finanzialisierung erheblich ausgeweitete Größe der Finanzmärkte in Betracht gezogen wird. Ohne Finanzmärkte von hypertrophen Ausmaßen hätte die Krise im Subprime-Sektor kaum zu schwersten Wirtschaftskrise seit den 1920er Jahre führen können. Nicht zuletzt sind jene kontinentaleuropäischen Ökonomien, die weniger finanzialisiert sind, vom Ausmaß der aktuellen Finanzkrise wesentlich geringer betroffen als die hochgradig finanzialisierten Ökonomien des angelsächsischen Kernlandes; wegen der nachfolgenden schweren Rezession in den westlichen Industrieländern können sich allerdings auch wenig finanzialisierte Ökonomien den Auswirkungen der Finanzkrise nicht völlig entziehen.

\section{Kapitalistische Vielfalt und die Finanzkrise im internationalen Vergleich}

\subsection{Varianz der Krisenanfälligkeit verschiedener Volkswirtschaften}

Aus einer vergleichenden Perspektive drängt sich die Frage auf, warum die Finanzkrise manche Volkswirtschaften und Sektoren stärker betrifft als andere. Offenkundig sind nicht alle Wirtschaften gleich von dieser Krise berührt, auch wenn das genaue Ausmaß der Wertberichtigungen erst in einigen Jahren feststehen wird. Ohne Zweifel gehören die hochgradig finanzialisierten Ökonomien der USA und Großbritanniens zu den am stärksten betroffenen Fällen, was sich insbesondere auf den britischen Inseln mittelfristig verheerend auswirken dürfte, angesichts der mit dem Ausbau des Finanzsektors einhergehenden Erosion der Industriebasis während der letzten Dekaden. In beiden Ländern kam es bereits frühzeitig zu einer Reihe von (Teil-)Verstaatlichungen von Banken sowie zu finanziell sehr umfangreichen Stützungsmaßnahmen der öffentlichen Hand; in den USA ging die Finanzkrise zudem mit einer erheblichen Konzentration im Finanzsektor einher.

Auch die kontinentaleuropäischen Ökonomien Deutschlands und Frankreichs sowie jene Japans sind von der Finanzkrise erheblich betroffen, wenn auch weniger schwer als die angelsächsischen Ökonomien. Trotzdem gab es eine Reihe von spektakulären Bankenzusammenbrüchen bzw. Fastzusammenbrüchen (IKB, Sachsen-LB, HypoReal, Dexia, Fortis) sowie von erheblichen Wertberichtigungen (z. B. Bayern LB, Deutsche Bank). Ein Vergleich der Abschreibungen mit der Größe des gesamten Finanzsektors zeigt jedoch, dass diese Volkswirtschaften relativ weniger betroffen sind als jene der angelsächsischen Länder. 
Abbildung 2: „,Abschreibungsliga”: Verluste durch die Finanzkrise pro Bank

\begin{tabular}{|c|c|c|c|}
\hline & Land & Unternehmen & $\begin{array}{l}\text { Gesamte Abschreibungen und } \\
\text { Verluste seit Januar } 2007 \\
\text { (Milliarden US \$) }\end{array}$ \\
\hline 1 & US & Citigroup & 60.8 \\
\hline 2 & US & Wachovia & 52.7 \\
\hline 3 & US & Merill Lynch & 52.2 \\
\hline 4 & US & Washington Mutual & 45.6 \\
\hline 5 & $\mathrm{CH}$ & UBS & 44.2 \\
\hline 6 & UK & HSBC & 27.4 \\
\hline 7 & US & Bank of America & 21.2 \\
\hline 8 & US & JPMorgan Chase & 18.8 \\
\hline 9 & US & Morgan Stanley & 15.7 \\
\hline 10 & GER & IKB Deutsche Industriebank & 14.3 \\
\hline 11 & UK & Royal Bank of Scotland & 13.8 \\
\hline 12 & US & Lehman Brothers & 13.8 \\
\hline 13 & GER & Deutsche Bank & 10.1 \\
\hline 14 & $\mathrm{CH}$ & Crédit Suisse & 10.1 \\
\hline 15 & US & Wells Fargo & 10 \\
\hline 16 & FRA & Crédit Agricole & 8.6 \\
\hline 17 & UK & Barclays & 7.5 \\
\hline 18 & CAN & $\begin{array}{l}\text { Canadian Imperial Bank of } \\
\text { Commerce }\end{array}$ & 7.1 \\
\hline 19 & BENELUX & Fortis & 6.9 \\
\hline 20 & GER & Bayerische Landesbank & 6.7 \\
\hline 21 & UK & HBOS & 6.6 \\
\hline 22 & NL & ING & 6.5 \\
\hline 23 & FRA & Société Générale & 6.4 \\
\hline 24 & JAP & Mizuho Financial Group & 6.1 \\
\hline & \multicolumn{2}{|l|}{ Weltweit } & 586.2 \\
\hline
\end{tabular}

Quelle: Financial Times

(http://www.ft.com/cms/s/0/e75aedae-20fe-11dd-a0e6-000077b07658,dwp uuid=698e638ee39a-11dc-8799-0000779fd2ac.html; 6.11. 2008). 
Zunächst relativ wenig betroffen von den unmittelbaren Auswirkungen der Finanzkrise blieben die meisten Schwellenländer (zum Beispiel Indien, Südafrika, verschiedene lateinamerikanische Staaten). Schwerwiegender sind hier eher die Folgen der nachfolgenden Rezession der westlichen Industrieländer, als Abnehmer von Rohstoffen und Industriegütern. Auch China sowie die nahöstlichen Erdölexporteure waren von der Finanzkrise zunächst in einigen Fällen spektakulär betroffen, insofern ihre Finanzmarktinstitutionen und Sovereign Wealth Funds in einer relativ frühen Phase der Krise die Schwäche der angelsächsischen Banken zum Erwerb erheblicher Anteile genutzt hatten und entsprechend erhebliche Verluste erlitten; insgesamt sind die Wertberichtigungen in diesen Ländern, verglichen zu jenen in den USA, jedoch begrenzt.

\subsection{Finanzialisierung und Vergleichende Kapitalismusforschung}

Um die Divergenz der Ausmaße der Finanzmarktkrise nach Ländern zu erklären, können wir auf das Theorieprogramm der Vergleichenden Kapitalismusforschung (»Comparative Capitalism«, Jackson/Deeg 2006) zurückgreifen. Im Rahmen dieses Forschungsprogramms sind in den letzten beiden Dekaden eine Vielzahl von Kapitalismustypologien entstanden, mit unterschiedlichen theoretischen Wurzeln in der Regulationstheorie (Hollingsworth/Boyer 1997, Amable 2003), dem Neomarxismus (Coates 2000) und dem Neoinstitutionalismus (Hall/Soskice 2001 a). Im Gegensatz zur Diskussion über fordistische und postfordistische (finanzialisierte) Produktionsregime wird hier die unterschiedliche Umsetzung solcher Entwicklungsphasen des (westlichen) Kapitalismus nach Ländern in den Vordergrund gestellt: »Der Fordismus war ein dominantes Produktionsregime, aber er wurde in unterschiedlichen Varianten in den einzelnen Ländern reproduziert « (Windolf 2005 b: 14). So wie der Fordismus nicht überall gleichmäßig umgesetzt wurde, gibt es auch Divergenzen bei der Umsetzung der Finanzialisierung, die sich zumindest in wesentlichen Zügen aus der theoretischen Perspektive der Vergleichenden Kapitalismusforschung erklären lassen (vgl. auch Seabrooke/Schwartz 2008).

Wenn an dieser Stelle auch nicht detailliert auf eine Gegenüberstellung von grundlegenden Merkmalen von koordinierten Ökonomien (zum Beispiel Deutschland) und liberalen Ökonomien (zum Beispiel die USA) sowie der Rolle des Finanzsektors in diesen Modellen eingegangen werden kann, wird die ungleich größere Wirkung der Finanzkrise auf liberale Ökonomien mühelos plausibel. In der angelsächsischen Variante des Kapitalismus nehmen die Kapitalmärkte die zentrale Rolle bei der Unternehmensfinanzierung ein und waren in den letzten Jahren ein erheblicher Wachstumsmotor. Diese Länder haben von der Finanzialisierung der letzten drei Dekaden besonders stark profitiert, sind jedoch nun auch vergleichsweise grundlegend von deren Konsequenzen betroffen. Allerdings sollte der Ausbruch dieser schwerwiegenden Finanzkrise nicht mit dem notwendigen Niedergang dieser Ökonomien verwechselt werden - ganz abgesehen davon, dass auch diese Ökonomien nicht immer finanzialisiert waren, verfügen insbesondere die USA mit ihrem großen - und von manchen 
Theorievarianten der Vergleichenden Kapitalismusforschung tendenziell ignorierten (vgl. z. B. Hall/Soskice 2001 b) - Staatssektor über einen wesentlichen Kompensationsfaktor.

Interessanter ist hingegen die Frage, warum auch die deutsche koordinierte Marktökonomie in einigen Fällen spektakulär von der Finanzkrise betroffen ist. Deutschland ist ein Spätstarter in der Finanzialisierung, erst die rot-grüne Regierung hat diesen Prozess durch eine Reihe von Gesetzgebungsinitiativen deutlich beschleunigt. Inzwischen lässt sich allerdings feststellen, dass die Finanzialisierung auch im »rheinischen Kapitalismus« einen ganz erheblichen Druck ausgeübt und diesen vielfältig erodiert hat, insofern die kurzfristige shareholder value-Orientierung in einem ganz erheblichen Spanungsverhältnis zu den langfristigen Geschäftsstrategien deutscher Unternehmen stehen kann. Diese - insbesondere am Kölner Max-Planck-Institut untersuchte -»Entbettung des rheinischen Kapitalismus « (Nölke 1998) wirkt allerdings eher langfristig und ist für die Turbulenzen der letzten Jahre vergleichsweise wenig einschlägig. So wird im Vergleich zur öffentlichen Diskussion über verschiedene Facetten der Finanzialisierung deutlich, dass dieser Prozess in Deutschland nach wie vor wesentlich weniger Fuß gefasst hat, als zum Beispiel in den Niederlanden, mit ihrer zentralen Bedeutung von privaten Pensionsfonds für die Altersversorgung (Engelen et al. 2008). Relevanter für die Auswirkungen der Finanzkrise ist allerdings, dass auch eine Reihe deutscher Banken die Auflösung der »Deutschland-AG«, insbesondere den Verkauf ihrer erheblichen Unternehmensbeteiligungen, zum Anlass genommen haben, sich ihrerseits erheblich auf den US-Finanzmärkten zu engagieren (vgl. van Treeck et al. 2007: 641).

Zudem hat auch hier wieder die politische Komponente des Finanzialisierungsprozesses eine große Rolle gespielt, insofern die - neben den privaten Banken maßgeblich von der Europäischen Kommission vorangetriebene - Abschaffung der Gewährsträgerhaftung (beschlossen 2001, in Kraft getreten 2005) das traditionelle Geschäftsmodell der deutschen Landesbanken durch den Wegfall von deren besonders günstigen Refinanzierungsmöglichkeiten fundamental in Frage gestellt hat, insbesondere angesichts der in Deutschland sehr ausgeprägten Konkurrenz im Bankensektor. Auf der Suche nach Möglichkeiten, trotz dieser Modifikation ihre häufig als zu niedrig kritisierten Eigenkapitalrenditen zu erhöhen und die in der Übergangsphase noch zugänglichen günstigen Refinanzierungsmöglichkeiten zu nutzen, begannen diese Banken, sich in erheblichem Maße an riskanten Verbriefungsgeschäften zu beteiligen (Braunberger 2007). Aus der Perspektive der Vergleichenden Kapitalismusforschung sind diese Finanzmarktaktivitäten deutscher Banken jedoch »artfremd « - sie passen nicht zu den anderen Institutionen, es gibt keine Komplementaritäten und auch die im Vergleich zu den angelsächsischen Banken wesentlich geringeren Erfahrungen werden dazu beigetragen haben, dass viele deutsche Großbanken in diesen Geschäften existenzbedrohende Verluste gemacht haben. Charakteristisch ist hingegen in diesem Zusammenhang, dass die deutschen Sparkassen und Genossenschaftsbanken, das für den deutschen Finanzsektor charakteristische, zu anderen Institutionen komplementäre und trotz der oben beschriebenen Erosionsprozesse stabile Merkmal (Hackethal/ Schmidt 2005), von der Finanzkrise relativ geringfügig betroffen sind, gerade weil 
sie sich an ihre etablierten Aktivitäten - etwa als Hausbanken des deutschen Mittelstands - hielten und sich relativ wenig an Finanzialisierungsprozessen beteiligten.

Das Theorieprogramm der Vergleichenden Kapitalismusforschung ist bisher nur in geringem Maße auf Länder außerhalb Westeuropas, Japans und der USA angewendet worden (vgl. Nölke/Vliegenthart 2009 zu Osteuropa). Trotzdem fällt es nicht schwer, aus dieser Perspektive die relativ geringen Auswirkungen der Finanzkrise - im Gegensatz zur nachfolgenden weltwirtschaftlichen Rezession - in den asiatischen Schwellenländern zu erklären, angesichts der relativ stark ausgeprägten staatlichen Restriktionen der Finanzmärkte dieser Staaten. Besonders wenig betroffen sind hier jene Staaten, die - wie die westlichen Industrieländer vor dem Kollaps des Bretton Woods-Systems - ihre Finanzsysteme nicht grundlegend liberalisiert haben, wie zum Beispiel Südafrika.

\section{Schlussfolgerungen}

Insgesamt hilft uns die Analyse der aktuellen Finanzkrise aus der FinanzialisierungsPerspektive sowie mit Hilfe des Theorieprogramms der Vergleichenden Kapitalismusforschung, die großen historischen Linien in der Fülle der Details der aktuellen Ereignisse $\mathrm{zu}$ identifizieren. Weiterhin erlaubt uns diese Theoriekombination auch die Entwicklung einer alternativen Perspektive, im Gegensatz zu einer Öffentlichkeit, die die Finanzkrise als US-Problem, Politikversagen oder als Scheitern einzelner Banker oder Politiker wahrnimmt. Dementsprechend legt die hier skizzierte IPÖPerspektive tendenziell andere Implikationen nahe, als der mainstream der Wirtschaftswissenschaften oder andere Theorieprogramme der IB: Aus der hier vorgestellten Sicht stellt die Finanzkrise der letzten Jahre sowie ihre zukünftige Überwindung weniger eine Frage der Koordination zwischen den G8 oder anderer kurzfristiger Reparaturmaßnahmen dar, sondern eher eine grundlegende Herausforderung zum Nachdenken über ein mögliches Ende der Epoche der Finanzialisierung, das auf diese Epoche möglicherweise folgende Wirtschaftsmodell und die in diesem Zusammenhang zu erwartenden Schwerpunktverschiebungen in der globalen politischen Ökonomie. Hier wartet eine große Forschungsagenda auf unsere Disziplin. Gerade die Verknüpfung der Finanzialisierungsperspektive mit dem Theorieprogramm der Vergleichenden Kapitalismusforschung würde die bisherige internationale Forschung zu dieser Thematik, die Finanzialisierung eher als aus der Perspektive einer kulturellen IPÖ oder als makroökonomisches Phänomen betrachtet, ergänzen, insbesondere durch einen stärkeren Fokus auf die politisch-institutionellen Ursachen und Konsequenzen der Finanzialisierung.

\section{Literatur}

Abdelal, Rawi 2007: Capital Rules: The Construction of Global Finance, Cambridge, MA. 
Allen, Franlklin/Carletti, Elena 2006: Mark-to-Market Accounting and Liquidity Pricing (CFS Working Paper 17), Frankfurt a. M.

Amable, Bruno 2003: The Diversity of Modern Capitalism, Oxford.

Andrews David M. (Hrsg.) 2008: Orderly Change: International Monetary Relations Since Bretton Woods, Ithaca, NY.

Arrighi, Giovanni 1994: The Long Twentieth Century: Money, Power, and the Origins of Our Times, London.

Best, Jacqueline/Paterson, Matthew (Hrsg.) 2009: Cultural Political Economy, London, i. E.

Bieling, Hans-Jürgen 2005: Finanzmarktintegration und transnationale Interessengruppen in der Europäischen Union, in: Eising, Rainer/Kohler-Koch, Beate (Hrsg.): Interessenpolitik in Europa, 179-226, Baden-Baden.

Blackburn, Robin 2008: The Subprime Crisis, in: New Left Review 50, 63-108.

Boyer, Robert 2000: Is a Finance-Led Growth Regime a Viable Alternative to Fordism? A Preliminary Analysis, in: Economy and Society 29: 1, 111-145.

Braunberger, Gerald 2007: Die Landesbanken suchen nach Geschäftsmodellen, in: Frankfurter Allgemeine Zeitung, 15.12.2007, 14.

Burn, Gary 1999: The State, the City and the Euromarkets, in: Review of International Political Economy 6: 2, 225-26.

Cerny, Philip G. 2008: Embedding Neoliberalism: The Evolution of a Hegemonic Pattern, in: The Journal of International Trade and Diplomacy 2: 1, 1-46.

Coates, David 2000: Models of Capitalism: Growth and Stagnation in the Modern Era, Cambridge.

Crotty, James 2002: The Effects of Increased Product Market Competition and Changes in Financial Markets on the Performance of Nonfinancial Corporations in the Neoliberal Era (PERI Working Paper 44), Amherst, MA.

Deutschmann, Christoph 2008: Der kollektive »Buddenbrooks-Effekt «. Die Finanzmärkte und die Mittelschichten (MPIfG Discussion Paper 5), Köln.

Emunds, Bernhard 2008: Wirtschaftsethik - Risiken, die niemand im Griff hat. Sozialethische Anmerkungen zur aktuellen Finanzmarktkrise, in: Herder-Korrespondenz 62: 9, 460-464.

Engelen, Ewald/Konings, Martijn/Fernandez, Rodrigo 2008: The Rise of Activist Investors and Patterns of Practical Responses: Lessons on Agency, in: Socio-Economic Review 6: 4, 611-636.

Epstein, Gerald A 2001: Introduction: Financialization and the World Economy, in: ders. (Hrsg): Financialization and the World Economy, Cheltenham, 3-16.

Erturk, Ismail/Froud, Julie/Solari, Stefano/Williams, Karel 2005: The Reinvention of Prudence: Household Savings, Financialisation and Forms of Capitalism (CRESC Working Paper 11), Manchester.

Erturk, Ismai/ Froud, Julie/Johal, Sukhdev/Leaver, Adam/Williams, Karel 2008: Financialization at Work: Key Text and Commentary, Milton Park.

Foster, John Bellamy 2008: The Financialization of Capital and the Crisis, in: Monthly Review 59: 11, 1-19.

Froud, Julie/Haslam, Colin/Sukhdev, Johal/Williams, Karel 2000: Shareholder Value and Financialization: Consultancy Promises, Management Moves, in: Economy and Society 29: $1,80-110$.

Hackethal, Andreas/Schmidt, Reinhard H. 2005: Strucural Change in the German Banking System? (Working Paper Series: Finance \& Accounting 147), Frankfurt a. M.

Hall, Peter A./Soskice, David (Hrsg.) 2001 a: Varieties of Capitalism: The Institutional Foundations of Comparative Advantage, Oxford.

Hall, Peter A./Soskice, David 2001 b: An Introduction to Varieties of Capitalism, in: Hall, Peter A./Soskice, David (Hrsg.) 2001 a, 1-68.

Helleiner, Eric 1994. States and the Reemergence of Global Finance: From Bretton Woods to the $1990 \mathrm{~s}$, Ithaca, NY. 
Hilferding, Rudolf 1910: Das Finanzkapital. Eine Studie über die jüngste Entwicklung des Kapitalismus, Wien.

Hollingsworth, James/Boyer, Robert: Contemporary Capitalism: The Embeddedness of Institutions, Cambridge.

Jackson, Gregory/Deeg, Richard 2006: How Many Varieties of Capitalism? Comparing the Comparative Institutional Analyses of Capitalist Diversity (MPIfG Discussion Paper 2), Köln.

Kädtler, Jürgen 2005: Finanzmärkte - Zur Soziologie einer organisierten Öffentlichkeit (SOFIMitteilungen 33), Göttingen.

Kädtler, Jürgen/Sperling, Hans Joachim 2001: Worauf beruht und wie wirkt die Herrschaft der Finanzmärkte auf die Ebene von Unternehmen? Oder: Taugt die Finanzialisierung als neue Software für die Autoindustrie? (SOFI-Mitteilungen 29), Göttingen.

Kindleberger, Charles 1978: Manias, Panics and Crashes: A History of Financial Crises, London.

Konings, Martijn 2008: The Institutional Foundations of US Structural Power in International Finance: From the Re-Emergence of Global Finance to the Monetarist Turn, in: Review of International Political Economy 15: 1, 35-61.

Krippner, Greta R. 2005: The Financialization of the American Economy, in: Socio-Economic Review 3: 2, 173-208.

Langley, Paul 2008: The Everyday Life of Global Finance: Saving and Borrowing in AngloAmerica, Oxford.

Lee Mudge, Stephanie 2008: What is Neo-Liberalism?, in: Socio-Economic Review 6: 4, 703-731.

Leyshon, Andrew/Nigel Thrift 2007: The Capitalization of Almost Everything: The Future of Finance and Capitalism, in: Theory, Culture \& Society 24: 7-8, 97-115.

Montgomerie, Johnna 2006: The Finanzialisation of the American Credit Card Industry, Competition and Change 10:3,301-319.

Nölke, Andreas 1998: Nichtkonventionelle Nichtmarktstrukturen bei der Unternehmensfinanzierung: Kapitalismustypen und die Auswirkungen der Globalisierung am Beispiel der institutionellen Investoren, in: Comparativ 8: 4, 45-62.

Nölke, Andreas/Perry, James 2007: The Power of Transnational Private Governance: Financialization and the IASB, in: Business and Politics 9: 3, Artikel 4.

Nölke, Andreas/Vliegenthart, Arjan 2009: Enlarging the Varieties of Capitalism: The Emergence of Dependent Market Economies in East Central Europe, in: World Politics 61: 4, i. E.

Orhangazi, Özgür 2007: Financialization and Capital Accumulation in the Non-Financial Corporate Sector: A Theoretical and Empirical Investigation of the U.S. Economy: 1973-2003 (PERI Working Paper 149), Amherst, MA.

Overbeek, Henk, Van Appeldoorn, Bastiaan/Nölke, Andreas (Hrsg.) 2007: The Transnational Politics of Corporate Governance Regulation, London.

Palley, Thomas I. 2007: Financialization: What It Is and Why It Matters? (Levy Economics Institute Working Paper 525), Washington, DC.

Perry, James/Nölke, Andreas 2006: The Political Economy of International Accounting Standards, in: Review of International Political Economy 13: 3, 559-586.

President of the United States of America 2008: Economic Report of the President, Washington, DC.

Schwartz, Herman M./Seabrooke, Leonard 2008: Varieties of Residential Capitalism in the International Political Economy: Old Welfare States and the New Politics of Housing, in: Comparative European Politics 6: 3, 237-261.

Stockhammer, Engelbert 2007: Some Stylized Facts on the Finance-Dominated Accumulation Regime (PERI Working Paper 142), Amherst, MA.

Useem, Peter 1996: Investor Capitalism: How Money Managers Are Changing the Face of Corporate America, New York, NY. 
Van Treeck, Till/Hein, Eckhard/Dünhaupt, Petra 2007: Finanzsystem und wirtschaftliche Entwicklung in den USA und in Deutschland im Vergleich. Eine makroökonomische Skizze (WSI Mitteilungen 12), Düsseldorf.

Windolf, Paul (Hrsg.) 2005 a: Finanzmarkt-Kapitalismus. Analysen zum Wandel von Produktionsregimen, Wiesbaden.

Windolf, Paul 2005 b: Einleitung »Die neuen Eigentümer«, in: Windolf, Paul (Hrsg.): Finanzmarkt-Kapitalismus. Analysen zum Wandel von Produktionsregimen, 516, Wiesbaden.

Windolf, Paul 2008: Eigentümer ohne Risiko, in: Zeitschrift für Soziologie 37: 6, 516-535. 